\title{
Estudio de los parámetros de freído sobre las propiedades fisicoquímicas de una botana hecha de harinas de maíz, chicharo y salvado de avena
}

Study of frying process parameters on the physicochemical properties of a snack made from corn flours, whole pea and oat bran

\author{
Enrique Arriola-Guevara1, Dafnne Monserrat Gudiño-García², Rogelio Prado-Ramírez², \\ Pedro Martín Mondragón-Cortez ${ }^{2}$, Rosa Isela Corona-González ${ }^{1}$, \\ Guadalupe María Guatemala-Morales ${ }^{2 *}$ (D)
}

${ }^{1}$ Universidad de Guadalajara, Centro Universitario de Ciencias Exactas e Ingenierías, Guadalajara Departamento de Ingeniería Química, , Jalisco - México

${ }^{2}$ Centro de Investigación y Asistencia en Tecnología y Diseño del Estado de Jalisco, A. C. Tecnología Alimentaria,

Guadalajara, Jalisco - México

*Corresponding Author: Guadalupe M. Guatemala-Morales, Centro de Investigación y Asistencia en Tecnología y Diseño del Estado de Jalisco, A. C. Tecnología Alimentaria, Avenida Normalistas, 800, Colinas de la Normal, 44270, Guadalajara, Jalisco - México, e-mail: guadisga@msn.com

Cite as: Arriola-Guevara, E., Gudiño-García, D. M., Prado-Ramírez, R., Mondragón-Cortez, P. M., CoronaGonzález, R. I., \& Guatemala-Morales, G. M. (2020). Study of frying process parameters on the physicochemical properties of a snack made from corn flours, whole pea and oat bran. Brazilian Journal of Food Technology, 23, e2018297. https://doi.org/10.1590/1981-6723.29718

\begin{abstract}
Resumen
El objetivo de este estudio fue evaluar el efecto de la temperatura y tiempo de freído sobre las propiedades fisicoquímicas: porcentaje de humedad, $\mathrm{H}$; cambio neto de color, $\Delta \mathrm{E}$; contenido de grasa, $\mathrm{G}$; índices de absorción, IAA; solubilidad en agua, ISA; densidad aparente, DA; y dureza, D, de un alimento frito tipo botana preparado con una mezcla de harinas de maíz nixtamalizado (HMN), harina de chícharo (HC) y salvado de avena (SA), hidratada al $47 \%$. El estudio mostró que $\mathrm{DA}$ y $\mathrm{H}$ disminuyeron, mientras que $\triangle \mathrm{E}$ e IAA se incrementaron con el aumento de la temperatura y el tiempo de freído. Los cambios en ISA y D resultaron estadísticamente no significativos. El análisis de componentes principales, mostró que el $83 \%$ de la variabilidad de los datos se puede explicar debido a dos componentes: temperatura (56\%) y tiempo (28\%). El análisis sensorial mostró que la botana elaborada a $165^{\circ} \mathrm{C}$ y $198 \mathrm{~s}$ obtuvo mayor aceptación, resultando un alimento con alto contenido de proteína y bajo contenido de grasa.
\end{abstract}

Palabras clave: Tiempo de freído; Harina de chícharo; Harina de maíz; Salvado de avena; Propiedades fisicoquímicas; Análisis de componentes principales.

\section{Abstract}

This work aimed to evaluate the effect of the frying time and temperature on the physicochemical properties: moisture percentage, $\mathrm{H}$; net color change, $\Delta \mathrm{E}$; fat content percentage, $\mathrm{G}$; water absorption index, WAl; water solubility index, WSI; bulk density, DA; and hardness, $\mathrm{D}$, of a fried snack made with a flour mixture of 
nixtamalized corn $(\mathrm{HMN})$, yellow pea $(\mathrm{HC})$, and oat bran (SA), hydrated to $47 \%$. The analysis showed that DA and $\mathrm{H}$ decreased, while $\Delta \mathrm{E}$, and WAl increased with the increase of time and temperature. WSI and $\mathrm{D}$ were not statistically significant. The principal component analysis showed that $83 \%$ of the variability of the data could be explained due to two components: temperature (56\%) and time (28\%). The sensory analysis showed better acceptance of the snack fried at $165^{\circ} \mathrm{C}$ and $198 \mathrm{~s}$, resulting in a food with high protein and low fat content compared to regular snacks.

Keywords: Frying time; Yellow pea flour; Corn flour; Oat bran; Physicochemical properties; Principal component analysis.

\section{Introducción}

La mayoría de las botanas elaboradas con maíz, aportan gran cantidad de carbohidratos; además, las grasas están presentes debido al proceso de freído al cual son sometidos este tipo de productos (Escobedo García, 2013).

Debido al auge que han tomado los productos naturales en los últimos tiempos, la producción de botanas busca obtener productos más nutritivos, con un mejor aporte de proteínas, calorías, fibra, ácidos grasos esenciales, vitaminas y minerales. Se han realizado, mediante las combinaciones con leguminosas, muchos intentos para elevar el valor nutritivo de la proteína en los productos a base de maíz (Lazou et al., 2011; Jacques-Fajardo et al., 2017).

El consumo de cereales y leguminosas en un solo alimento aumenta la calidad de la proteína consumida debido a la complementación de aminoácidos que se produce (Granito et al., 2003). El consumo de ambas especies en la dieta podría incrementarse con el desarrollo de alimentos de fácil consumo atractivos al consumidor (Sozer et al., 2017).

El chícharo contiene altos niveles de proteínas, fibras dietéticas, hidratos de carbono complejos, isoflavonas y ácido fólico y es bajo en grasa y sodio. La mayoría de estos componentes también están asociados con beneficios para la salud, como las proteínas con efectos hipocolesterolémicos, y las isoflavonas que ayudan en la prevención de la osteoporosis y ciertos tipos de cáncer (Nayak et al., 2011; Jacques-Fajardo et al., 2017).

El salvado de avena puede contener niveles por arriba del 15\% del producto, éste es la capa más externa comestible del grano de avena y se produce durante el molido de avenas, o avena arrollada, para separar la harina resultante por tamizado. Es un subproducto rico en fibra, que contiene las capas externas fragmentadas del grano junto con cantidades variables del endospermo almidonado. En contraste con el salvado de trigo, el salvado de avena es rico en fibra soluble en agua y pobre en celulosa y lignina (Jacques-Fajardo et al., 2017). El mayor inconveniente relacionado con la incorporación de algún tipo de fibra en los alimentos, es el efecto que produce sobre sus propiedades físicas y sensoriales. Algunos cambios en el sabor, la palatabilidad, la apariencia y la textura pueden no ser aceptados por los comerciantes y consumidores (Pavlovich-Abril et al., 2012).

El objetivo de este trabajo fue evaluar el efecto de la temperatura y tiempo de freído sobre las propiedades fisicoquímicas de un alimento frito, tipo botana, preparado a base de una mezcla de harinas de maíz nixtamalizado (HMN), de chícharo entero (HC) y salvado de avena (SA), hidratada al $47 \%$. Asimismo, la realización de un análisis sensorial ayudó a identificar las condiciones del proceso para obtener la botana con mayor aceptación. 


\section{Metodología}

\subsection{Materiales}

Se utilizó chícharo lizo amarillo entero (Pisum sativum) para la HC y SA en presentación de costal, adquirido en un mercado local. La HMN fue de la línea especial tipo BFR de la marca MINSA ${ }^{\circledR}$. En la fritura se utilizó aceite vegetal comestible marca Bakers \& Chefs $^{\circledR}$.

La $\mathrm{HC}$ es el producto resultante del escaldado de los granos, seguido de secado con aire y la subsecuente molienda. El proceso de escaldado se realizó siguiendo la metodología de Alasino et al. (2008), por inmersión en agua caliente a $100{ }^{\circ} \mathrm{C}$ durante $1,5 \mathrm{~min}$, seguido del enfriamiento inmediato por inmersión en agua a $20^{\circ} \mathrm{C}$.

El chícharo seco y el salvado de avena, se sometieron a un proceso de molienda usando un molino de martillos (Weber Bros ${ }^{\circledR}$, malla No. 8).

\subsection{Análisis proximales}

En las harinas y el producto final frito, se determinó humedad (925.10, AOAC), proteínas $(\mathrm{N}$ x 6,25) (991.20, AOAC), cenizas (923.03, AOAC), grasa (923.05, AOAC), fibra cruda (962.09, AOAC), carbohidratos (986.25, AOAC), fibra total, soluble e insoluble (991.43, AOAC) y grasa (920.39, AOAC). El porcentaje de humedad $(\% \mathrm{H})$ en cada uno de los tratamientos del producto frito, se determinó con un analizador de humedad AND ${ }^{\circledR}$ MX-50. Se pesaron aproximadamente $5 \mathrm{~g}$ de cada tratamiento de producto frito.

\subsection{Determinación del tamaño de partícula de harinas, DTP.}

La DTP de las harinas se realizó en un equipo Rotap ${ }^{\circledR}$ RX-29 con un conjunto de mallas 40, 60, 80, 100, 120, 150. Por cada malla se determinó el peso retenido de harina y luego se calculó el porcentaje en relación al total de la muestra con la Ecuación 1 (Gaytán-Martínez et al., 2011).

$D T P=\frac{W_{1} D_{1}+W_{2} D_{2}+W_{3} D_{3}+W_{4} D_{4}+W_{5} D_{5}+W_{6} D_{6}}{T S}$

donde: $W_{l-6}$ es el peso de las partículas retenidas en cada tamiz; $D_{l-6}$ tamaño de malla de cada tamiz (abertura) y $T S$ es el tamaño de la muestra total.

\subsection{Determinación de Índice de absorción de agua, IAA e Índice de solubilidad de agua, ISA}

La determinación de IAA e ISA de las harinas y los tratamientos de producto frito, se realizaron siguiendo el método de Jacques-Fajardo et al. (2017). Se mezclaron 2,5 g de muestra con $25 \mathrm{~mL}$ de agua destilada, agitándose en un equipo Roto-Shake Genie ${ }^{\circledR}$ durante $30 \mathrm{~min}$, para después centrifugarse (4 $000 \mathrm{rpm}, 30 \mathrm{~min}, 25^{\circ} \mathrm{C}$ ) en un equipo Hettich ${ }^{\circledR}$ Universal $320 \mathrm{R}$. El sobrenadante se decantó y se secó en estufa a $105^{\circ} \mathrm{C}$ durante $24 \mathrm{~h}$. El IAA (Ecuación 2) se calculó como el peso de sedimento o gel obtenido después de la eliminación del sobrenadante por unidad de peso de los sólidos originales como base seca y expresado como g/g muestra. El ISA (Ecuación 3) fue obtenido por la división del peso de los sólidos secos recuperados por evaporación entre el peso de la muestra. Los resultados se expresan en porcentaje.

$$
\begin{aligned}
& I A A=\frac{\text { Pesodel gel }(g)}{\text { Peso muestra seca }(g)} \\
& I S A=\frac{\text { Peso de sólidos secos }}{\text { Peso muestra seca }} \times 100
\end{aligned}
$$




\subsection{Color ( $L^{*}, a^{*}$ y *b) y cambio neto de color $(\Delta E)$}

Se realizaron mediciones de color en las harinas y en cada uno de los tratamientos de producto frito, utilizando las coordenadas de color CIE L*a*b* (Commission Internationale de I'Eclairage, 1976) con un colorímetro MiniScan HunterLab ${ }^{\circledR}$ equipado con el iluminante CIE $\mathrm{D}_{65}(6500 \mathrm{~K})$. El colorímetro se calibró con una placa negra de porcelana ( $0 \%$ de reflectancia) y una placa blanca ( $100 \%$ de reflectancia). Para los tratamientos de producto frito se determinó el $\Delta \mathrm{E}$, comparado con el parámetro promedio de las harinas $\left(L_{p}^{*}=22,39, a_{p}^{*}=0,49, b^{*}{ }_{p}=5,17\right)$ con la Ecuación 4.

$$
\Delta E=\sqrt{\left(L_{p}^{*}-L_{i}^{*}\right)^{2}+\left(a_{p}^{*}-a_{i}^{*}\right)^{2}+\left(b_{p}^{*}-b_{i}^{*}\right)^{2}}
$$

donde el subíndice $p$ corresponde a la lectura estándar de la placa de calibración, y el subíndice $i$ corresponde a cada uno de los tratamientos.

\subsection{Densidad real $\left(\rho_{R}\right)$ y densidad aparente $\left(\rho_{A}\right)$ de harinas}

La $\rho_{R}$ de las harinas se determinó con el método del picnómetro sugerido por Koocheki et al. (2007). Se utilizó tolueno como disolvente de densidad conocida. Se determinó el peso del sólido (harina) $\left(M_{l}\right)$, el peso del picnómetro lleno de tolueno hasta la señal de enrase $\left(M_{2}\right)$ y el peso del picnómetro con el disolvente hasta la señal de enrase y el sólido $\left(M_{3}\right)$. La densidad real se calculó con las Ecuaciones 5 y 6.

$$
\begin{aligned}
& \rho_{R}=\frac{M_{1}}{V} \\
& V=\frac{M_{1}+M_{2}-M_{3}}{\rho_{\text {Tolueno }}}
\end{aligned}
$$

La $\rho_{A}$ y la ${ }_{\varphi}$ de las harinas se determinaron con el método de Koocheki et al. (2007). Una porción de harina $\left(W_{2}\right)$ se añadió a un cilindro volumétrico previamente pesado $\left(W_{l}\right)$ y el volumen se leyó como $V_{l}$. La densidad aparente se calculó con la Ecuación 7 y la porosidad con la Ecuación 8.

$$
\begin{aligned}
& \rho_{A}=\frac{W_{2}-W_{I}}{V_{I}} \\
& \varphi=1-\frac{\rho_{A}}{\rho_{R}}
\end{aligned}
$$

\subsection{Densidad aparente del producto frito, DA}

La DA se calculó con la medición de las dimensiones reales de los productos fritos (Hoyos et al., 2017). La densidad de las muestras se calculó en $\mathrm{g} / \mathrm{cm}^{3}$ con la Ecuación 9.

$$
D A=\frac{M}{\pi(\varnothing / 2)^{2} l}
$$

donde: el diámetro de la partícula es $(\varnothing)$ y la longitud $(l)$ y M es la masa de la muestra.

\subsection{Formulación y preparación}

La preparación de la masa se realizó manualmente; la formulación final utilizada fue de $32 \%$ de HMN, $16 \%$ de HC, $10 \%$ de SA y $42 \%$ de agua. Con contenido de humedad en base seca de $12,10,4 \%$, para HMN, HC y SA respectivamente.

Los churros fueron moldeadas con un embutidor de acero inoxidable, con una placa intercambiable con cuatro orificios de $6,75 \mathrm{~mm}$ de diámetro interno cada uno. El proceso de freído se realizó en una freidora Frymaster $^{\circledR}$ con capacidad de $25 \mathrm{~L}$. 


\subsection{Análisis de perfil textura de masa, (TPA)}

El TPA de la masa, se basó en los métodos sugeridos por Almeida \& Rooney (1996). Se utilizó un texturómetro Texture Analyser ${ }^{\mathbb{R}}$ TAX-T2 y las pruebas se efectuaron a temperatura ambiente $\left(25 \pm 2{ }^{\circ} \mathrm{C}\right)$. Se utilizaron muestras cilíndricas preparadas de la siguiente manera: un molde de acero inoxidable de forma cilíndrica con dimensiones internas de $18 \mathrm{~mm}$ de diámetro y $16 \mathrm{~mm}$ de altura; la masa se dejó reposar durante 15 minutos, dándosele forma laminar con un rodillo de madera y cortándose las muestras con el molde de acero inoxidable. Las muestras se sometieron a dos ciclos de compresión de 50\%, con relación a la altura original, con una velocidad de $1 \mathrm{~mm} \mathrm{~s}^{-1}$ teniendo un tiempo de espera entre los dos ciclos de $5 \mathrm{~s}$.

\subsection{Análisis de Dureza, (D) en producto frito}

El análisis de dureza, (D) de cada tratamiento de los productos fritos, se realizó con un texturómetro Texture Analyser ${ }^{\circledR}$ TAX-T2 con una punta de forma cónica y con una velocidad de prueba de $1 \mathrm{~mm} \mathrm{~s}^{-1}$. Las muestras se sometieron a dos ciclos de compresión del $50 \%$ con un tiempo de espera de $5 \mathrm{~s}$ (Aguilar-Palazuelos et al., 2012).

\subsection{Diseño experimental y análisis estadístico}

El diseño de experimentos para el proceso de freído se resolvió con Statgraphics ${ }^{\circledR}$. Se trata de un diseño factorial $2^{2}\left(150{ }^{\circ} \mathrm{C}-90 \mathrm{~s}, 150{ }^{\circ} \mathrm{C}-180 \mathrm{~s}, 180{ }^{\circ} \mathrm{C}-180 \mathrm{~s}\right.$ y $\left.180{ }^{\circ} \mathrm{C}-90 \mathrm{~s}\right)$ central compuesto más estrella $\left(144{ }^{\circ} \mathrm{C}-135 \mathrm{~s}, 165^{\circ} \mathrm{C}-198 \mathrm{~s}, 186{ }^{\circ} \mathrm{C}-135 \mathrm{~s}\right.$ y $\left.165^{\circ} \mathrm{C}-72 \mathrm{~s}\right)$ con 3 puntos centrales $\left(165{ }^{\circ} \mathrm{C}-135 \mathrm{~s}\right)$, en el que se estudia el efecto de 2 factores: temperatura y tiempo en 11 tratamientos. Las variables de respuesta analizadas fueron $\% \mathrm{H}$, los parámetros de color $L^{*}, a^{*}, b^{*}$ y $\Delta \mathrm{E}, \% \mathrm{G}$, IAA e ISA en agua, DA y D.

Para la correlación entre las propiedades fisicoquímicas y su relación con las condiciones de freído, se empleó el análisis de componentes principales (ACP) (Statgraphics Centurion, versión 16.2).

\subsection{Análisis sensorial}

El análisis sensorial de aceptación se realizó con 200 sujetos no entrenados y son consumidores habituales o potenciales. Se utilizó una escala hedónica de 5 puntos (5 - me gusta mucho a 1 - me disgusta mucho) para apariencia y sabor. El estudio se dividió en dos partes; menores y mayores de 18 años. Se seleccionaron las 3 muestras con menor porcentaje de humedad $\left(180{ }^{\circ} \mathrm{C}-180 \mathrm{~s}-3,10 \%\right.$, $\left.165^{\circ} \mathrm{C}-198 \mathrm{~s}-2,85 \%, 186^{\circ} \mathrm{C}-135 \mathrm{~s}-4,40 \%\right)$.

\section{Resultados}

\subsection{Caracterización de las harinas}

Con la caracterización de las harinas fue posible comparar la afectación en las propiedades fisicoquímicas de la botana frita sometida a los diferentes tratamientos.

En la Tabla 1 se muestran los resultados obtenidos de la caracterización de las harinas utilizadas. El tamaño de partícula promedio de la mezcla de las tres harinas fue de $0,188 \mathrm{~mm}$. La HMN presentó el mayor porcentaje de partículas retenidas en la malla de $0,380 \mathrm{~mm}$, seguido de las mallas de $0,229 \mathrm{~mm}$ y $0,140 \mathrm{~mm}$ lo cual indica la presencia de partículas gruesas y finas. Por su parte el SA presentó el mayor porcentaje de partículas retenidas en la malla de $0,380 \mathrm{~mm}$, lo cual se debe a la presencia de partículas gruesas. La HC presentó el porcentaje más alto en el fondo de los tamices, esto indica la presencia de partículas finas, ya que pasaron la malla de $0,104 \mathrm{~mm}$. La fracción fina es la responsable de la mayor parte de la absorción de agua y el desarrollo de viscosidad durante el mezclado. Por su parte, la fracción gruesa reduce el exceso de suavidad, o la formación de burbujas de aire de gran tamaño durante el cocimiento o el proceso de freído (García Jiménez \& Vázquez, 2016). 
Los valores obtenidos para el IAA de la HMN se encuentran entre los rangos reportados por Billeb de Sinibaldi \& Bressani (2001) (3,06-3,53 g gel/g harina) y Bressani et al. (2001) (3,41-4,02 g gel/g harina) para harina de maíz. Los valores de IAA de SA y HC, como era de esperarse, son menores a los reportados para harina de maíz. Mientras mayor daño exista en la estructura del almidón, la harina tenderá a absorber una mayor cantidad de agua (Contreras Jiménez et al., 2014). En relación al valor obtenido de ISA para HMN, este se encuentra dentro del rango que reporta Bressani et al. (2001) (4,77-7,00\%) para harinas comerciales. Mientras que el SA se encontró dentro del rango que reporta Billeb de Sinibaldi \& Bressani (2001) (3,22 a 4,72\%) para harina de maíz. Finalmente, el valor de ISA para HC reportado en este trabajo, es mayor a lo que reporta la literatura, lo cual podría deberse a la presencia de partículas finas en la harina.

Tabla 1. Propiedades fisicoquímicas de HMN, SA y HC.

\begin{tabular}{cccc}
\hline \multirow{2}{*}{ Parámetro } & \multicolumn{3}{c}{ Valores } \\
\cline { 2 - 4 } & HMN & SA & HC \\
\hline DTP $(\mathrm{mm})$ & 0,187 & 0,250 & 0,152 \\
\hline IAA (g/g muestra) & $3,48 \pm 0,09$ & $2,16 \pm 0,04$ & $2,29 \pm 0,12$ \\
\hline ISA $(\%)$ & $5,04 \pm 0,06$ & $4,42 \pm 0,16$ & $0,97 \pm 0,15$ \\
\hline$\rho_{A}(\mathrm{~g} / \mathrm{mL})$ & $0,50 \pm 0,10$ & $0,41 \pm 0,00$ & $1,74 \pm 0,0,62$ \\
\hline$\rho_{R}(\mathrm{~g} / \mathrm{mL})$ & $1,00 \pm 0,14$ & $1,83 \pm 0,53$ & $0,46 \pm 0,15$ \\
\hline$\varphi^{\prime}(-)$ & $0,49 \pm 0,17$ & $0,76 \pm 0,06$ & $49,28 \pm 2,21$ \\
\hline Color & & & $2,22 \pm 0,19$ \\
\hline$L^{*}(-)$ & $56,25 \pm 1,12$ & $17,81 \pm 0,89$
\end{tabular}

$\mathrm{HMN}=$ harina de maíz nixtamalizado; $\mathrm{SA}=$ salvado de avena; $\mathrm{HC}=$ harina de chícharo; $\mathrm{DTP}=$ tamaño de la partícula; IAA = índice de absorción e agua; ISA = índice de solubilidad en agua; $\rho_{A}=$ densidad aparente; $\rho_{R}=$ densidad real; $\varphi=$ porosidad y color CIE L*a*b*. Los valores indican el promedio \pm desviación estándar.

El ISA refleja la severidad del proceso, que va aumentando debido a modificaciones en el almidón causadas por molienda y condiciones de tratamiento. Wang et al. (2012), menciona que los factores que influye en la solubilidad son el tamaño del granulo, que facilita la entrada del agua por los espacios intermoleculares, y la presencia de mayor contenido de amilopectina.

La HC presentó mayor contenido de proteínas que la HMN, lo cual confirma que la proporción de proteínas en las leguminosas representa el doble de proteínas que en los cereales (Bressani \& Elías, 1980; Todorov, 1988). Las tres materias primas mostraron ser una rica fuente de carbohidratos. El SA confirmó tener un alto contenido de fibra $(14,27 \%$ en peso) de acuerdo a lo resultados de Falcón et al. (2011). La Tabla 2 muestra los resultados obtenidos del análisis proximal de las harinas utilizadas.

Tabla 2. Contenido proximal de HMN, SA y HC.

\begin{tabular}{cccc}
\hline Determinación & \multicolumn{3}{c}{ \% en peso } \\
\cline { 2 - 4 } & HMN & SA & HC \\
\hline Humedad & 7,18 & 8,98 & 9,04 \\
\hline Proteínas (N x 6,25) & 8,51 & 9,07 & 18,45 \\
\hline Cenizas & 1,34 & 1,89 & 2,30 \\
\hline Grasas (Extracto etéreo) & 3,37 & 6,21 & 67,15 \\
\hline Carbohidratos totales & 79,60 & 69,85 & 13,93 \\
\hline Fibra cruda & 12,05 & 14,40 & 0,36 \\
\hline Fibra dietética insoluble & 12,74 & 15,18 & 13,27 \\
\hline Fibra dietética soluble & $<0,10$ & $<0,10$ & 14,27 \\
\hline Fibra dietética total & 12,74 & 15,18 & \\
\hline
\end{tabular}

$\mathrm{HMN}=$ harina de maíz nixtamalizado; $\mathrm{SA}=$ salvado de avena; $\mathrm{HC}=$ harina de chícharo. 


\subsection{Propiedades de la masa}

Las propiedades texturales de la masa de maíz, dependen de la capacidad que tiene el almidón para retener agua (Rodríguez et al., 2005). Se han reportado valores de D, cohesividad y resiliencia de masas fresca (MFMN) y de harina $100 \%$ de maíz nixtamalizado (MHMN) hidratadas al 58\%. Para dureza, cohesividad y resiliencia de MFMN se han reportado valores de 14,50 $\pm 0,754,0,192 \pm 0,008$ y $0,034 \pm 0,003$ respectivamente; y para MHMN valores de $19,30 \pm 0,465,0,159 \pm 0,006$ y $0,038 \pm 0,001$ respectivamente (Gasca-Mancera \& Casas-Alencáster, 2007).

Los valores promedio y la desviación estándar de las propiedades mecánicas de alimentos mediante análisis de TPA obtenidos para la masa de la mezcla preparada, fueron de 19,53 $\pm 1,098,0,15 \pm 0,002 \mathrm{y}$ $0,04 \pm 0,001$ respectivamente. Los valores para estos parámetros se encuentran dentro de los valores obtenidos por Gasca-Mancera \& Casas-Alencáster (2007), observándose que la adición de HC y SA no afectan las propiedades mecánicas de la HMN.

\subsection{Propiedades fisicoquímicas de los productos fritos}

\subsubsection{Contenido de humedad}

Durante el proceso de freído ocurre una rápida deshidratación, llegándose hasta un contenido de humedad del 1,5-2,5\% (Almeida \& Rooney, 1996). El análisis estadístico para \% $\mathrm{H}$ del producto frito mostró diferencias significativas $(p \leq 0,05)$ en los factores temperatura y tiempo de freído en el análisis de varianza ANOVA (Tabla 3).

Tabla 3. Análisis de varianza ANOVA para \% $\mathrm{H}$.

\begin{tabular}{cccccc}
\hline Factor & $\begin{array}{c}\text { Suma de } \\
\text { Cuadrados }\end{array}$ & Gl & Cuadrado Medio & Razón-F & Valor-P \\
\hline A: Temperatura & 51,2553 & 1 & 51,2553 & 33,91 & 0,0006 \\
\hline B: Tiempo & 127,245 & 1 & 127,245 & 84,18 & 0,0000 \\
\hline AB & 1,26563 & 1 & 1,26563 & 0,84 & 0,3906 \\
\hline Error total & 10,5805 & 7 & 1,5115 & & \\
\hline Total (corr.) & 190,346 & 10 & &
\end{tabular}

La Figura 1, muestra como el \% $\mathrm{H}$ disminuye con el incremento de temperatura y tiempo de freído. El \% $\mathrm{H}$ varió de 15,90-2,0\% (Tabla 4), valores similares a los obtenidos para botanas formuladas 50:50 de harina de maíz y amaranto de $1,81 \%$, para botanas elaboradas con $100 \%$ harina de maíz nixtamalizado de 3,82\% y para algunos productos comerciales en un intervalo de 2,35-4,1\% reportados por Escobedo-García (2013).

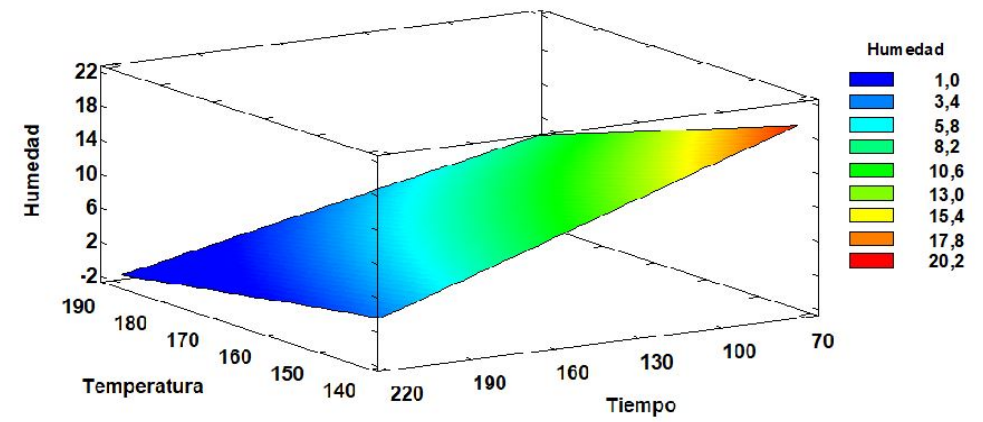

Figura 1. Gráfica de superficie de respuesta estimada para la humedad de la botana hecha a base de harinas de maíz, chícharo y salvado de avena. 


\subsubsection{Análisis del color}

Para los parámetros de color $L^{*}, a^{*}, b^{*}$, los análisis estadísticos mostraron diferencias significativas $(p \leq 0,05)$ en el efecto combinado de temperatura y tiempo de freído. El parámetro $a^{*}$ también mostró diferencias significativas $(p \leq 0,05)$ en el factor temperatura. El $\Delta \mathrm{E}$ no mostró efectos significativos. Se observó que los valores $a^{*}$ y $b^{*}$ disminuyen con el incremento de la temperatura y aumentan con el incremento del tiempo de freído.

$\mathrm{El}$ valor $L^{*}$ mostró un comportamiento inverso a los otros parámetros de color evaluados. El $\Delta \mathrm{E}$ aumenta con el incremento de tiempo de freído. Los valores obtenidos de los parámetros $a^{*}(4,72 \pm 0,67)$ y $b^{*}(15,50 \pm 2,00)$ son positivos, y les corresponden coloraciones rojizas y amarillas, respectivamente. Para $L^{*}$, se obtuvieron valores mayores alrededor de $24,41 \pm 0,98$, lo cual indica que se trata de un alimento ligeramente obscuro. Los valores de $\Delta \mathrm{E}$ se presentan en la Tabla 4. El grado de oscurecimiento de un producto frito es atribuible principalmente a la formación de compuestos producto de las reacciones de Maillard y caramelización (Fellows, 2000; Gökmen \& Şenyuva, 2007).

\subsubsection{Contenido de grasa}

El análisis estadístico para \% $\mathrm{G}$ mostró diferencias significativas $(p \leq 0,05)$ en el factor tiempo. Se observó que el contenido de grasa, presente en la botana, es menor a bajas temperaturas y tiempos cortos de freído (Tabla 4). La industria maneja un intervalo entre el 22 y el 28\% de grasa en producto terminado (Lucas et al., 2012). Algunos productos comerciales en sus formulaciones reportan un intervalo de 28,15 a 32,9\% de grasa (Escobedo García, 2013).

\subsubsection{Análisis de IAA e ISA}

Los resultados del análisis de varianza ANOVA para las variables de respuesta IAA e ISA, mostraron diferencias significativas $(p \leq 0,05)$ en los factores de temperatura y tiempo para IAA. El ISA del producto frito no resultó estadísticamente significativo y presentó valores similares a los de la masa cruda, lo cual indica que no hubo daño considerable a la estructura de las moléculas de fibra, almidón o proteínas, que generarán mayor presencia de compuestos solubles. La Tabla 4 muestras los resultados obtenidos en este trabajo. Se observó que el IAA incrementa con el aumento de temperatura y tiempo, lo cual podría deberse a la gelatinización del almidón. Tanto el IAA como el ISA dependen de la disposición de las moléculas de amilosa y amilopectina en los gránulos de almidón (Moreno et al., 2012).

\subsubsection{Densidad aparente}

El análisis estadístico para DA mostró diferencias significativas $(p \leq 0,05)$ en el factor temperatura. Se observó que los valores menores de este parámetro fueron obtenidos a altas temperaturas (Tabla 4). Mientras más bajo es el valor, el producto tendrá mejores características. En un estudio de evaluación de las propiedades físicas de la masa de las donas durante la fritura profunda a diferentes temperaturas, Gómez-Daza (2014), evidenció el efecto del tiempo y la temperatura de freido sobre la calidad física, textural y química del alimento en donde se encuentra implicita DA.

\subsubsection{Análisis de dureza}

Para D, el análisis estadístico no mostró diferencias significativas $(p \leq 0,05)$ para los factores temperatura y tiempo. Se observó que D incrementa con el aumento de temperatura y la disminución del tiempo de freído. La fuerza de dureza o punto de quiebre de los productos se usa para medir la crujencia de un producto frito (Shyu \& Hwang, 2001). Botanas elaboradas a base de maíz blanco gigante nixtamalizado frito en aceite (oleína) de palma a $180{ }^{\circ} \mathrm{C}$, por un tiempo de 8 a 13 minutos hasta que la botana alcanzó un 
color ligeramente dorado reportan un intervalo de D de $38 \mathrm{~N}$ a $58 \mathrm{~N}$ (Boluarte Alarcón et al., 2018). Por otra parte Yuksel et al. (2017) produjeron botanas de maíz con pan añejo en polvo y harina de trigo. El valor de dureza de las muestras de chips de maíz se vio significativamente afectado por la adición del pan añejo, las muestras de control fritas entre $170{ }^{\circ} \mathrm{C}$ y $190{ }^{\circ} \mathrm{C}$ por un tiempo entre 50 a $60 \mathrm{~s}$, obtuvieron valores de dureza que variaron entre 104 a $252 \mathrm{~N}$, muy por encima de los valores obtenidos en este trabajo (Tabla 4), lo que muestra la dependencia de ésta propiedad, sobre la composición, procedencia y variedad de la materia prima utilizada, así como también de los parámetros del proceso.

Tabla 4. Propiedades fisicoquímicas de los productos fritos.

\begin{tabular}{cccc}
\hline \multirow{2}{*}{ Parámetro } & \multicolumn{3}{c}{ Valores } \\
\cline { 2 - 4 } & Mínimo & Máximo & Promedio \pm Desviación estándar \\
\hline$\% \mathrm{H}$ & $2,85\left(165^{\circ} \mathrm{C}-90 \mathrm{~s}\right)$ & $15,90\left(165^{\circ} \mathrm{C}-72 \mathrm{~s}\right)$ & $8,55 \pm 4,30$ \\
\hline$\% \mathrm{G}$ & $19,41\left(165^{\circ} \mathrm{C}-72 \mathrm{~s}\right)$ & $27,45\left(165^{\circ} \mathrm{C}-198 \mathrm{~s}\right)$ & $23,67 \pm 2,40$ \\
\hline $\mathrm{IAA}(\mathrm{g} / \mathrm{g}$ muestra $)$ & $4,32\left(165^{\circ} \mathrm{C}-72 \mathrm{~s}\right)$ & $5,93\left(186^{\circ} \mathrm{C}-135 \mathrm{~s}\right)$ & $5,32 \pm 0,49$ \\
\hline $\mathrm{ISA}(\%)$ & $7,41\left(150{ }^{\circ} \mathrm{C}-90 \mathrm{~s}\right)$ & $15,22\left(144{ }^{\circ} \mathrm{C}-135 \mathrm{~s}\right)$ & $11,34 \pm 2,79$ \\
\hline $\mathrm{DA}\left(\mathrm{g} / \mathrm{cm}^{3}\right)$ & $0,54\left(186^{\circ} \mathrm{C}-135 \mathrm{~s}\right)$ & $0,90\left(144{ }^{\circ} \mathrm{C}-135 \mathrm{~s}\right)$ & $0,64 \pm 0,12$ \\
\hline $\mathrm{D}(\mathrm{N})$ & $19,07\left(144^{\circ} \mathrm{C}-135 \mathrm{~s}\right)$ & $39,76\left(180{ }^{\circ} \mathrm{C}-90 \mathrm{~s}\right)$ & $23,16 \pm 4,00$ \\
\hline
\end{tabular}

\subsection{Análisis sensorial}

Los resultados del análisis sensorial mostraron mayor preferencia en sabor y apariencia hacia el producto frito a $165{ }^{\circ} \mathrm{C}-198 \mathrm{~s}$ (Figura 2). La población de estudio se conformo por 50\% adultos y 50\% menores de 18 años, de los cuales 66 fueron mujeres adultas, 34 hombres adultos, 57 niñas y 43 niños, tanto mujeres $(61,5 \%)$ como hombres de los dos grupos de sujetos no entrenados mostraron preferencia por el producto frito a estas condiciones de operación. Este tratamiento, presentó menor contenido de humedad $(2,85 \%)$, densidad $\left(0,538 \mathrm{~g} / \mathrm{cm}^{3}\right)$ y dureza $(19,260 \mathrm{~N})$; y mayor porcentaje de grasa $(27,45 \%)$ comparada con las otras dos seleccionadas para este estudio.
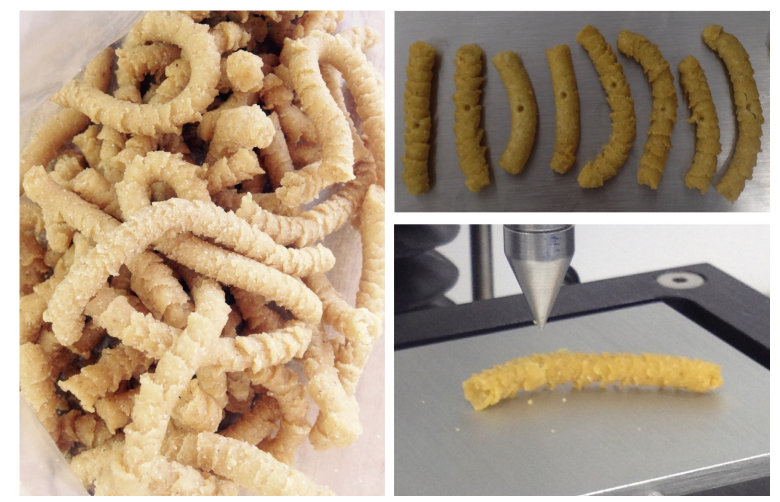

Figura 2. Apariencia de la botana elaborada a $165^{\circ} \mathrm{C}$ y $198 \mathrm{~s}$ con harinas de maíz, chícharo y salvado de avena.

El producto frito a $180{ }^{\circ} \mathrm{C}-180 \mathrm{~s}$, fue el segundo preferido en sabor y apariencia por el grupo de mayores de 18 años; y por sabor fue el tercer preferido sólo para el grupo de menores de 18 años. Este tratamiento presentó mayor valor para DA en comparación con las otras muestras seleccionadas. El producto frito a $186^{\circ} \mathrm{C}-135 \mathrm{~s}$ fue el segundo preferido en sabor para el grupo de menores de 18 años y el tercer preferido para el grupo de mayores de 18 años por su sabor y apariencia. Este tratamiento presentó el mayor valor de \% $\mathrm{H}$ comparado con las otras muestras seleccionadas. Las mujeres de los dos grupos calificaron con mayores puntajes los productos fritos seleccionados. En la Tabla 5 se muestran algunas propiedades fisicoquímicas de las muestras seleccionadas para el análisis sensorial. 
Tabla 5. Propiedades fisicoquímicas para el análisis sensorial.

\begin{tabular}{|c|c|c|c|c|c|}
\hline \multicolumn{2}{|c|}{ Proceso de freído } & \multicolumn{4}{|c|}{ Parámetro } \\
\hline Temperatura $\left({ }^{\circ} \mathrm{C}\right)$ & Tiempo (s) & $\% \mathrm{H}$ & $\% \mathrm{G}$ & DA $\left(\mathrm{g} / \mathrm{cm}^{3}\right)$ & $\mathrm{D}(\mathrm{N})$ \\
\hline 180 & 180 & 3,10 & 23,72 & 0,546 & 20,85 \\
\hline 165 & 198 & 2,85 & 27,45 & 0,538 & 19,26 \\
\hline 186 & 135 & 4,4 & 24,25 & 0,538 & 21,94 \\
\hline
\end{tabular}

$\mathrm{H}=$ humedad; $\mathrm{G}=$ grasa; $\mathrm{DA}=$ densidad aparente del produto frito; $\mathrm{D}=$ dureza en Newtons.

En la Tabla 6 se muestran los resultados obtenidos del análisis proximal de dicho tratamiento y los reportados por Escobedo García (2013) para botanas comerciales. Se observó que el producto frito de este trabajo presenta mayor contenido de proteínas y fibra; y menor contenido de grasas con relación a los valores presentados por las botanas comerciales mexicanas.

Tabla 6. Comparación del contenido proximal del producto frito.

\begin{tabular}{cccc}
\hline $\begin{array}{c}\text { Determinación } \\
\text { (\% en peso) }\end{array}$ & $\begin{array}{c}\text { Producto frito } \\
\text { (este trabajo) }\end{array}$ & $\begin{array}{c}{ }^{*} \mathbf{M C 1} \\
\text { (Maíz, amaranto y trigo) }\end{array}$ & $\begin{array}{c}\text { *MC2 } \\
\text { (Maíz, amaranto, trigo, } \\
\text { soya y ajonjolí) }\end{array}$ \\
\hline Proteínas (N x 6,25) & 9,43 & 8,25 & 7,55 \\
\hline Cenizas & 1,33 & 0,5 & 0,79 \\
\hline Grasas (Extracto etéreo) & 26,94 & 32,9 & 28,15 \\
\hline Carbohidratos totales & 58,13 & 52,5 & 59,35 \\
\hline Fibra cruda & 3,16 & - & - \\
\hline Fibra dietética insoluble & 3,98 & - & - \\
\hline Fibra dietética soluble & 0,20 & - & - \\
\hline Fibra dietética total & 4,18 & 1,75 & 1,81 \\
\hline
\end{tabular}

*Marcas comerciales (MC) (Escobedo García, 2013).

\subsection{Análisis de componentes principales, ACP}

Se realizó un ACP sobre las variables temperatura, tiempo, \% H, \% G, IAA y las variables relacionadas con la textura; DA, D, y Diámetro; para analizar el aporte de cada variable evaluada en cada componente (Figura 3). Generalmente, los primeros componentes principales (CP1 y CP2) explican la mayor varianza entre los datos utilizados.

Para el análisis, las variables de color $\left(L^{*}, a^{*}, b^{*}\right.$ y $\left.\Delta \mathrm{E}\right)$ e ISA fueron descartadas, debido a que no presentaron efectos significativos. El valor del índice de Kaiser-Meyer-Olkin fue de 0,68, y es estadísticamente aceptable. Los dos primeros componentes principales (CP1 y CP2) explicaron el 83,15\% de la varianza total. Estos componentes tuvieron valores eigen mayores o iguales que 1,0. El CP1 explicó el $55,59 \%$ de la varianza total y tiene mayor correlación con las variables tiempo, $\% \mathrm{H}$, IAA, DA y diámetro. El CP2 explicó el $27,57 \%$ de la varianza total y se correlaciona con las variables temperatura, $\%$ G y D.

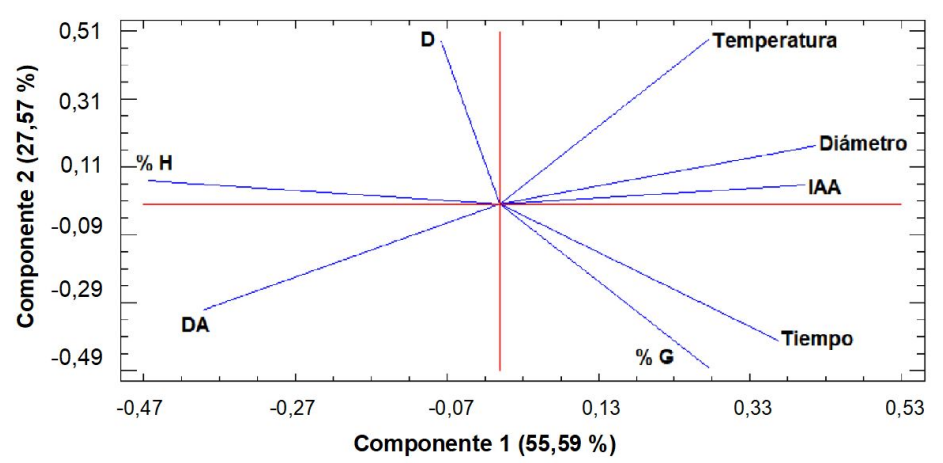

Figura 3. Gráfica de ACP que describe la variación y la correlación entre las diferentes condiciones de freído, los parámetros del proceso y las propiedades del producto frito. 


\section{Conclusiones}

La botana frita a base de harina de maíz nixtamalizada, salvado de avena y harina de chícharo, e hidratada al 47\%, mostró que la temperatura y el tiempo de freído son claves para la calidad fisicoquímica de producto final. El análisis de componentes principales, mostró que el $83 \%$ de la variabilidad de los datos se puede explicar debido a dos componentes, donde el $56 \%$ es debido a la temperatura y el $28 \%$ al efecto del tiempo.

Los porcentajes de humedad disminuyeron en el producto en función del aumento de la temperatura y el tiempo de freído. Se observó que el porcentaje de grasa presente en la botana fue menor a bajas temperaturas y tiempos cortos de freído. Los valores de Índice de solubilidade mostraron ligeros cambios durante los distintos tratamientos de freído, lo cual fue atribuida a la baja generación de compuestos solubles durante el tratamiento. El Índice de absorción de agua se incrementó en función del tiempo y temperatura de freído, lo cual fue atribuido a la gelatinización del almidón durante los tratamientos. La dureza del producto se incrementó con el aumento de la temperatura y la disminución del tiempo de freído. El análisis sensorial mostró que la preferencia de los consumidores fue por la botana frita de baja densidade aparente $\left(0,538 \mathrm{~g} / \mathrm{cm}^{3}\right)$ y dureza $(19,260 \mathrm{~N})$, la cual fue elaborada a $165^{\circ} \mathrm{C}$ y 198 segundos.

\section{Agradecimientos}

Los autores agradecen los apoyos dados por el Centro de Investigación y Asistencia en Tecnología y Diseño del Estado de Jalisco, A. C., y a la Universidad de Guadalajara.

\section{Referencias}

Aguilar-Palazuelos, E., Zazueta-Morales, J. J., Harumi, E. N., \& Martínez-Bustos, F. (2012). Optimization of extrusion process for production of nutritious pellets. Food Science and Technology, 32(1), 34-42. http://dx.doi.org/10.1590/S010120612012005000005

Alasino, M. C., Andrich, O. D., Sabbag, N. G., Costa, S. C., De La Torre, M. A., \& Sánchez, H. D. (2008). Panificación con harina de arvejas (Pisum sativum) previamente sometidas a inactivación enzímática. Archivos Latinoamericanos de Nutricion, 58(4), 397-402. PMid:19368302.

Almeida, H. D., \& Rooney, L. W. (1996). Avances en la manufactura y calidad de productos de maíz nixtamalizado. Industrie Alimentari, 18, 4-13.

Billeb de Sinibaldi, A. C., \& Bressani, R. (2001). Características de cocción por nixtamalización de once variedades de maíz. Archivos Latinoamericanos de Nutricion, 51(1), 86-94. PMid:11515238.

Boluarte Alarcón, G. I., Dionisio Fernández, C. P., \& Cisneros, F. H. (2018). Efecto del tipo de agente de pelado, tiempo de cocción y contenido de humedad en la calidad de snacks fritos de maíz blanco gigante (Zea mays). Revista de la Sociedad Química del Perú, 84(1), 157-171.

Bressani, R., \& Elías, L. G. (1980). Nutritional value of legume crops for humans and animals. In R. J. Summerfield \& A. H. Bunting (Eds.), Advances in legume science (pp. 135-155). Great Britain. Royal Botanic Gardens.

Bressani, R., Turcios, J. C., Reyes, L., \& Mérida, R. (2001). Caracterización física y química de harinas industriales nixtamalizadas de maíz de consumo humano en América Central. Archivos Latinoamericanos de Nutricion, 51(3), $309-313$. PMid:11791485.

Commission Internationale de l'Eclairage - CIE. (1976). Oficial recommendations on uniform color spaces: Color difference equations and metric color terms: Colorimetry (Vol. 15, No. 2). Paris: CIE.

Contreras-Jiménez, B., Morales-Sánchez, E., Reyes-Vega, M. L., \& Gaytánmartínez, M. (2014). Propiedades funcionales de harinas de maíz nixtamalizado obtenidas por extrusión a baja temperatura. Journal of Food, 12(3), 263-270. http://dx.doi.org/10.1080/19476337.2013.840804

Escobedo García, J. I. (2013). Desarrollo de una botana complementada con amaranto (Amaranthus hypochondriacus) para aumentar su calidad nutrimental (Disertación). Facultad de Estudios Superiores Cuautitlán, Universidad Nacional Autónoma de México, Estado de México. Recuperado el 18 de noviembre de 2018, de http://132.248.9.195/ptd2013/enero/0687435/Index.html

Falcón, M. R., Barrón, J. M., Romero, A. L., \& Domínguez, M. F. (2011). Efecto adverso en la calidad proteica de los alimentos de dietas con alto contenido de fibra dietaria. Revista Chilena de Nutrición, 38(3), 369-375. http://dx.doi.org/10.4067/S071775182011000300012 
Fellows, P. (2000). Frying technology. In P. Fellows (Ed.), Food processing technology: Principles and practice (2nd ed., Cap. 17, pp. 355-360). Cambridge: Woodhead Publishing.

García Jiménez, A., \& Vázquez, C. L. (2016). Masa y harina de maiz nixtamalizado. Investigación y Desarrollo en Ciencia y Tecnología de Alimentos, 1(2), 78-82.

Gasca-Mancera, J. C., \& Casas-Alencáster, N. B. (2007). Adición de harina de maíz nixtamalizado a masa fresca de maíz nixtamalizado: Efecto en las propiedades texturales de masa y tortilla. Revista Mexicana de Ingeniería Química, 6(3), 317-328.

Gaytán-Martínez, M., Figueroa, J. D. C., Morales-Sánchez, E., Vázquez-Landaverde, P. A., \& Martínez-Flores, H. E. (2011). Physicochemical properties of masa and corn tortilla made by ohmic heating. African Journal of Biotechnology, 10(71), 1602816036. http://dx.doi.org/10.5897/AJB11.1820

Gökmen, V., \& Şenyuva, H. Z. (2007). Acrylamide formation is prevented by divalent cations during the Maillard reaction. Food Chemistry, 103(1), 196-203. PMid:26054281. http://dx.doi.org/10.1016/j.foodchem.2006.08.011

Gómez-Daza, J. C. (2014). Evaluación de las propiedades físicas de la masa de las donas durante la fritura profunda a diferentes temperaturas: Análisis crítico. Revista UGCiencia, 20, 87-95.

Granito, M., Torres, A., \& Guerra, M. B. (2003). Desarrollo y evaluación de una pasta a base de trigo, maíz, yuca y frijol. Interciencia, 28, 372-379.

Hoyos, J. L., Villada, H. S., Fernández, A., \& Ortega-Toro, R. (2017). Parámetros de calidad y metodologías para determinar las propiedades físicas de alimentos extruidos para peces. Información Tecnológica, 28(5), 101-114. http://dx.doi.org/10.4067/S0718-07642017000500012

Jacques-Fajardo, G. E., Prado-Ramírez, R., Arriola-Guevara, E., Pérez Carrillo, E., Espinosa-Andrews, H., \& Guatemala Morales, G. M. (2017). Physical and hydration properties of expanded extrudates from a blue corn, yellow pea and oat bran blend. Lebensmittel-Wissenschaft + Technologie, 84, 804-814. http://dx.doi.org/10.1016/j.Iwt.2017.06.046

Koocheki, A., Razavi, S. M. A., Milani, E., Moghadam, T. M., Abedini, M., Alamatiyan, S., \& Izadkhah, S. (2007). Physical properties of watermelon seed as a function of moisture content and variety. International Agrophysics, 21, 349-359.

Lazou, A., Krokida, M., Zogzas, N., \& Karathanos, V. (2011). Lentil-based snacks: Structural and textural evaluation. Procedia Food Science, 1, 1593-1600. http://dx.doi.org/10.1016/j.profoo.2011.09.236

Lucas, A. J. C., Quintero, C., Vasco, V. D., Leal, J. F., \& Mosquera, A. (2012). Evaluación de los parámetros de calidad de chips en relación con diferentes variedades de plátano (Musa paradisíaca L.). Revista Lasallista de Investigacion, 9(2), 65-74.

Moreno, M. M., Rodríguez Sandoval, E., \& Sepúlveda Valencia, J. U. (2012). Evaluación de las propiedades físicas y texturales del buñuelo. Revista Lasallista de Investigacion, 9(2), 112-121.

Nayak, B., Berrios, J. D. J., Powers, J. R., \& Tang, J. (2011). Effect of extrusion on the antioxidant capacity and color attributes of expanded extrudates prepared from purple potato and yellow pea flour mixes. Journal of Food Science, 76(6), 874-883. PMid:22417485. http://dx.doi.org/10.1111/j.1750-3841.2011.02279.x

Pavlovich-Abril, A., Rouzaud-Sández, O., Torres, P., \& Robles-Sánchez, R. M. (2012). Cereal bran and wholegrain as a source of dietary fibre: Technological and health aspects. International Journal of Food Sciences and Nutrition, 63(7), 882-892. PMid:22486424. http://dx.doi.org/10.3109/09637486.2012.676030

Rodríguez, E., Fernández, A., \& Ayala, A. (2005). Reología y textura de masas: Aplicaciones en trigo y maíz. Revista Ingeniería e Investigación, (57), 72-78.

Shyu, S., \& Hwang, L. S. (2001). Effects of processing conditions on the quality of vacuum fried apple chips. Food Research International, 34(2-3), 133-142. http://dx.doi.org/10.1016/S0963-9969(00)00141-1

Sozer, N., Holopainen-Mantila, U., \& Poutanen, K. (2017). Traditional and new food uses of pulses. Cereal Chemistry, 94(1), 66-73. http://dx.doi.org/10.1094/CCHEM-04-16-0082-FI

Todorov, N. A. (1988). Cereals, pulses and oilseeds. Livestock Production Science, 19(1-2), 47-95. http://dx.doi.org/10.1016/0301-6226(88)90086-3

Wang, Y., Zhang, M., \& Mujumdar, A. S. (2012). Influence of green banana flour substitution for cassava starch on the nutrition, color, texture and sensory quality in two types of snacks. Food Science and Technology, 47(1), 175-182.

Yuksel, F., Karaman, S., Gurbuz, M., Hayta, M., Yalcin, H., Dogan, M., \& Kayacier, A. (2017). Production of deep-fried corn chips using stale bread powder: Effect of frying time, temperature and concentration. Lebensmittel-Wissenschaft + Technologie, 83(1), 235-242. http://dx.doi.org/10.1016/j.lwt.2017.04.076 